\title{
A functional assay to study the pathogenicity of CHD7 protein variants encountered in CHARGE syndrome patients
}

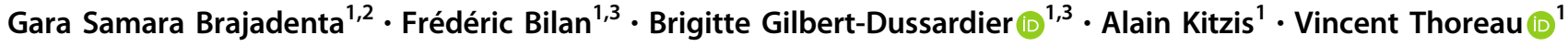

Received: 5 February 2019 / Revised: 4 June 2019 / Accepted: 25 June 2019 / Published online: 9 July 2019

(c) The Author(s), under exclusive licence to European Society of Human Genetics 2019

\begin{abstract}
CHARGE syndrome is a rare genetic disease characterized by numerous congenital abnormalities, mainly caused by de novo alterations of the $C H D 7$ gene. It encodes a chromodomain protein, involved in the ATP-dependent remodeling of chromatin. The vast majority of $C H D 7$ alterations consists in null alleles like deletions, nonsense substitutions or frameshift-causing variations. The aim of this study was to develop a biological test of CHD7 protein, to study the impact upon protein functionality of rare allelic variants in the CHD7 gene that elicits changes in the amino acid sequence. Using an expression vector encoding CHD7, three amino acid substitutions and one five-amino acid insertion were generated via site-directed mutagenesis. Then CHD7 proteins, either wild-type (WT) or variants, were overexpressed in HeLa cell line. Protein expression was highlighted by western blot and immunofluorescence. We then used real-time RT-PCR to study CHD7 functionality by evaluating the transcript amounts of five genes whose expression is regulated by CHD7 according to the literature. These reporter genes are 45S rDNA, SOX4, SOX10, ID2, and MYRF. We observed that, upon WT-CHD7 expression, the reporter gene transcriptions were downregulated, whereas the four variant alleles of CHD7 had no impact. This suggests that these alleles are not polymorphisms because the variant proteins appeared nonfunctional. Therefore, these variations can be considered as disease-causing of CHARGE syndrome.
\end{abstract}

\section{Introduction}

CHARGE syndrome (CS; OMIM 214800) is a rare genetic disease characterized by the multiple congenital anomalies in variable combinations [1, 2]. Pagon et al. coined the term CHARGE, an acronym summarizing six cardinal clinical features of the disorder (ocular Coloboma, Heart disease, choanal Atresia, growth and/or development $\underline{\text { Retardation, }}$

Supplementary information The online version of this article (https:// doi.org/10.1038/s41431-019-0465-7) contains supplementary material, which is available to authorized users.

$\triangle$ Vincent Thoreau

vincent.thoreau@univ-poitiers.fr

1 Neurovascular Unit and Cognitive Disorders (EA-3808 NEUVACOD), Université de Poitiers, Poitiers, France

2 Department of Medical Biology, Division of Human Genetics, Faculty of Medicine, University of Swadaya Gunung Jati, Cirebon, Indonesia

3 Department of Genetics, Centre Hospitalier Universitaire de Poitiers, Poitiers, France
Genito-urinary defects and/or hypogonadism, Ear anomalies and/or deafness) [3]. The diagnosis has subsequently been refined, as the combinations of major and minor criteria [4]. The incidence of CHARGE syndrome was estimated from $1 / 8500$ to $1 / 12,000$ live births [5]. In 2004, molecular abnormalities in patients with CHARGE syndrome were identified using CGH (comparative genomic hybridization) array and FISH (fluorescent in situ hybridization). They concluded that the CHD7 gene (OMIM 608892) is responsible for the most cases of CHARGE syndrome [6]. A large majority of $\mathrm{CHD7}$ allelic variants occurs de novo; however, rare familial occurrences have been described, with dominant inheritance but with heterogeneous phenotypes within families [7]. Regardless of the case, haploinsufficiency mechanism is responsible for the pathology. Some cases of significant clinical overlap with CHARGE syndrome, such as 22q11.2 deletion syndrome, Kallmann syndrome and Omenn-like syndrome, have also shown CHD7 alterations [8-10].

CHD7 protein belongs to the chromodomain helicase DNA-binding (CHD) protein family, which is a highly conserved group of nuclear proteins that exhibit ATPdependent chromatin remodeling activity [11]. Among 
them, CHD7 is involved in the fine-tuning of gene transcription in the early steps of the development of various tissues [12]. Indeed, CHD7 appeared to bind various chromatin sites. If the binding pattern is cell-dependent, it always matches with the chromatin fragments recognized by a specific subset of methylated H3 histones [13]. CHD7 localizes in the nucleoplasm and it is also found in the nucleolus, where it positively regulates the expression of the 45S ribosomal RNA precursor [14].

For $78 \%$ of patients, the expression of one CHD7 allele is abolished following so-called truncating alterations, e.g., nonsense and frameshift eliciting the appearance of a premature termination codon, and $11 \%$ of patients have splice site alterations. By contrast, only $8 \%$ of the $C H D 7$ variants found in patients are missense [7]. However, the deleterious consequence of a single amino acid substitution may be challenging to ascertain, hindering genetic counseling. Computational tools have been refined to predict putative deleterious effects of missense variants [15]. Moreover, DNA methylation signature was recently proposed as a diagnosis tool for disorders involving proteins that attend the epigenetic machinery $[16,17]$.

However, no straightforward functional assay is available for $\mathrm{CHD} 7$ protein variants. A biochemical method was described to test in vitro the function of the CHD7 protein: first, the authors purified CHD7 protein expressed in insect cells using a baculovirus vector. Then, they used these recombinant proteins to study in vitro their chromatin remodeling properties [18]. However, the complexity of this system is incompatible with routine use.

In this paper, we propose a simple assay, in which CHD7 protein variants are expressed in HeLa cell line. Then, the impact upon the transcription of several genes, whose expression is controlled by CHD7 protein according to the literature, is evaluated by qRT-PCR. In order to discriminate nonfunctional proteins and to identify likely disease-causing variants, the effect of the expression of CHD7 proteins exhibiting amino acid substitutions or insertion found in patients was compared with the one of wild-type CHD7.

\section{Materials and methods}

\section{Patients and molecular genetics data}

The index cases were referred to our laboratory because of clinical features characteristic of CHARGE syndrome according to Verloes's updated criteria [4]. CHD7 variation screening was realized as previously described [19, 20]. Nucleotide RefSeq CHD7 NM_017780.3 and exon numbering NG_007009.1 were used. CHD7 variants affecting its function were classified according to the American
College of Medical Genetics recommendations [21]. To investigate the potential impact of missense nucleotide changes upon CHD7 protein, we have used 10 software embedded in the VarSome tool (https://varsome.com/) and PolyPhen-2 (http://genetics.bwh.harvard.edu/pph2/). To establish the potential impact on the splicing mechanism, missense variants were studied by using Human Splicing Finder (HSF) software (http://www.umd.be/HSF3/).

Patient harboring c.5944 $\mathrm{G}>\mathrm{T}$ or p.(Gly1982Trp): the female index patient ( 9 years old) presented with a typical form of CS (notably semicircular canal hypoplasia, atresia of the choanae, tetralogy of fallot, and typical cup-shaped ears). This variant was found in her affected brother (intellectual deficiency, unilateral deafness, and cryptorchidism) and was not found in her mother (her father, presenting with unilateral deafness, refused $C H D 7$ molecular analysis) (Supplementary Fig. 1). Nevertheless, her paternal uncle (53 years old) who also harbors this variation presented with a typical CS form (complete clinical description previously published) [20]. Gly1982Trp variation affects the SANT (switching-defective protein 3, adaptor 2, nuclear receptor corepressor, transcription factor IIIB) domain of the protein and is predicted pathogenic by all software. The variation is not found in any public database. Taken together, these data sustain a class 3 (uncertain significance) variation. It could be noticed that HSF predicted no significant impact upon splicing mechanism.

Patient harboring c.6955 C > T (rs121434341) or p. (Arg2319Cys): this young male baby (9 months old) presented with an atypical CS form with a bilateral choanae atresia, a right semicircular canal hypoplasia and typical dysplastic ears. Ophthalmologic, cardiac, and abdominal examinations were normal. This missense variant is predicted pathogenic by all software and arose de novo. It is reported one time as pathogenic in ClinVar (https://www. ncbi.nlm.nih.gov/clinvar/), five times in the CHD7 database (https://chd7.org) and is not reported in control database like Exome Variant Server (EVS) (http://evs.gs.washington. edu/EVS/) or gnomAD (http://gnomad.broadinstitute.org/). Moreover, another variant affecting the same amino acid (p. Arg2319Ser) is also reported twice as pathogenic in ClinVar and twice as VUS (variant of unknown significance) in CHD7 database. Taken together, these data are consistent with p.(Arg2319Cys) belonging to class 5 (disease-causing) variation. HSF predicted no significant impact upon splicing mechanism.

Patient harboring c.3905 T > C or p.(Leu1302Pro): this male patient (13 years old) presented with an atypical form of CS (detailed clinical description previously published) [21]. This variant localized in Helicase $\mathrm{C}$ domain is predicted pathogenic by all software and arose de novo. It was reported once as pathogenic in the CHD7 database and was not reported in the control database like EVS or gnomAD. 
All these data are consistent with a class 5 (disease-causing) variation. HSF predicted no significant impact upon splicing mechanism.

We reported our findings in ClinVar database and these variants can be viewed using the following accession numbers: SCV000899279 (c.5944 G > T), SCV000899230 (c.6955 C>T), and SCV000899280 (c.3905 T>C). We also posted CS patients and their corresponding variants in CHD7 database under the following references: pending.

We have previously described patients displaying c.5405-17 G > A substitution (rs794727423), which lies in a recurrent hotspot of variations in intron 25 of CHD7 [22]. We have shown by the minigene assay that it elicits a splicing defect, potentially leading to the synthesis of a protein harboring a five-amino acid insertion, $p$. (His1801_Gly1802insAspGlyHisGlyThr).

As functional variant controls, we used c.2680 A>G (rs377662366) or p.(Thr894Ala), and c.6478 G $>$ A (rs61753399) or p.(Ala2160Thr). Both are classified as benign in ClinVar.

The positions in CHD7 protein of the amino acid changes that we studied are displayed in Fig. 1.

\section{Plasmids and site-directed mutagenesis}

The pCI-neo vector encoding untagged wild-type CHD7 protein is a generous gift from Cynthia Bartels and Peter Scacheri (Case Western Reserve University, Cleveland, Ohio, USA). Nucleic acid substitutions were generated using the QuickChange XL Site-Directed Mutagenesis Kit (Agilent Technologies, Santa Clara, California, USA). To generate a five-amino acid insertion, we applied a modified mutagenesis protocol [23]. The DNA sequences of the oligonucleotides (Eurogentec, Seraing, Belgium) are listed in Supplementary Table 1. Plasmid DNA was purified using the QIAprep Spin Miniprep or Maxiprep Kits (Qiagen, Hilden, Germany) according to the supplier's instructions. Sequence modifications in individual clones were verified through plasmid sequencing on both strands using the ABI PRISM Big Dye Terminator Cycle Sequencing Ready

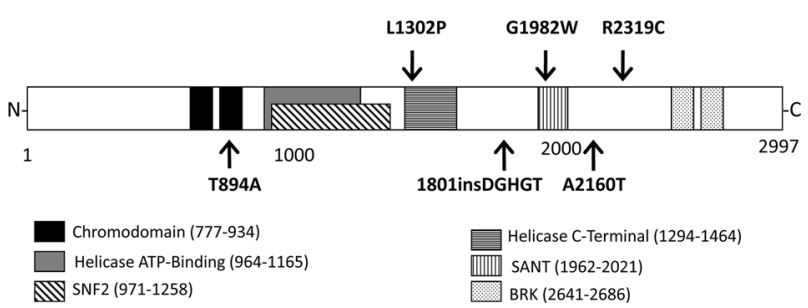

Fig. 1 Schematic organization of CHD7 protein. CHD7 consists in 2997 amino acids. This representation shows the distribution of CHD7 protein domains. The estimated domain boundaries were determined in http://www.ebi.ac.uk/interpro/protein/Q9P2D1. Arrows indicate the positions of the amino acid sequence variations that we studied
Reaction Kit (Applied Biosystems, Foster City, California, USA). Reactions were run on an ABI PRISM 310 automatic sequencer (Applied Biosystems).

\section{Cell culture and transfection}

HeLa cells were cultured under an atmosphere of $5 \% \mathrm{CO}_{2}$ at $37^{\circ} \mathrm{C}$ in Dulbecco's Modified Eagles Medium (DMEM, Invitrogen, Carlsbad, California, USA), supplemented with $10 \%$ fetal bovine serum (Invitrogen) and $1 \%$ penicillin-streptomycin $(10,000 \mathrm{U} / \mathrm{mL}$, Invitrogen). For transitory transfection, cells were seeded at a density of $4.10^{5}$ cells per well in 6 -well culture plates, or $8.10^{5}$ cells per $60 \mathrm{~mm}$ culture dish, and were incubated overnight at $37^{\circ} \mathrm{C}$ to obtain $70-80 \%$ confluence. Transfection was performed with $5 \mu \mathrm{L}$ of Lipofectamine 2000 (Invitrogen) mixed with $4 \mu \mathrm{g}$ of plasmid DNA, or with $10 \mu \mathrm{L}$ of Lipofectamine 2000 and $8 \mu \mathrm{g}$ of plasmid DNA, respectively.

\section{Antibodies}

The following primary antibodies were used in this study: rabbit monoclonal antibody anti-CHD7 (clone D3F5, Cell Signaling, Danvers, Massachusetts, USA); mouse monoclonal antibodies anti- $\beta$-actin (clone AC-74, Sigma-Aldrich, St. Louis, Missouri, USA), and anti-nucleolin (C23, clone MS-3, Santa Cruz Biotechnology, Dallas, Texas, USA). Peroxidase-linked secondary antibodies for western blot were obtained from Sigma-Aldrich: goat anti-mouse IgG (Fc-specific) and goat anti-rabbit IgG (whole molecule). For fluorescent imaging, we used RRX-conjugated goat antimouse $\operatorname{IgG}(\mathrm{H}+\mathrm{L})$ and FITC-conjugated donkey antirabbit $\operatorname{IgG}(\mathrm{H}+\mathrm{L})$ (Jackson ImmunoResearch Laboratories, Cambridgeshire, UK).

\section{Western blot analysis}

Forty-eight hours after transfection, cells were solubilized in lysis buffer containing $10 \mathrm{mM}$ Tris- $\mathrm{HCl} \mathrm{pH} 7.5,1 \%$ Nonidet P-40, $0.5 \%$ deoxycholate with protease inhibitor cocktail Complete (Roche Applied Science, Penzberg, Germany) and $2 \mathrm{mM}$ AEBSF (Sigma-Aldrich). Cell lysate homogenization, SDS-PAGE, and western blot were performed as previously described [24].

\section{Immunofluorescence}

Cells were cultured on glass coverslips, transfected and incubated at $37^{\circ} \mathrm{C} 48 \mathrm{~h}$ before staining. Then they were fixed in $3.7 \%$ paraformaldehyde and permeabilized in PBS containing $0.5 \%$ Triton X-100 (Sigma-Aldrich). Unspecific antigen sites were blocked with PBS containing $0.5 \%$ bovine serum albumin (Sigma-Aldrich). Cells were 

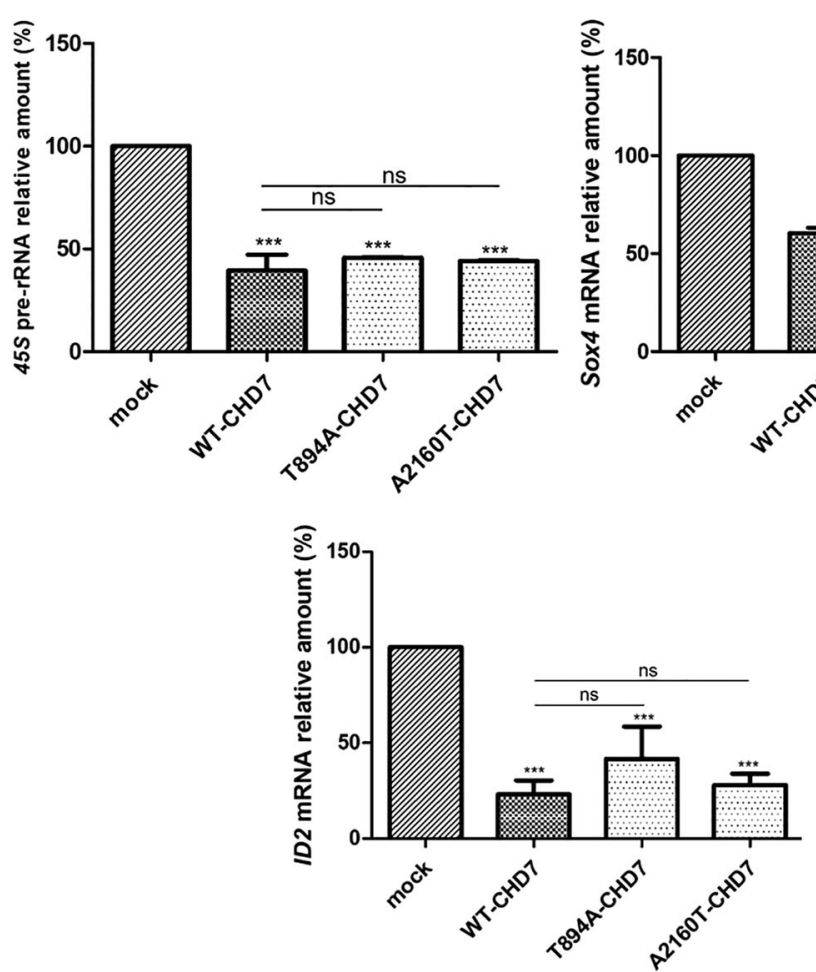

Fig. 2 Impact of functional $C H D 7$ alleles expression upon the transcription of five reporter genes. After transfection of wild type or variant forms of CHD7, we performed qRT-PCR to evaluate the impact upon the transcription of genes regulated by CHD7: $45 \mathrm{~s}$ rDNA,
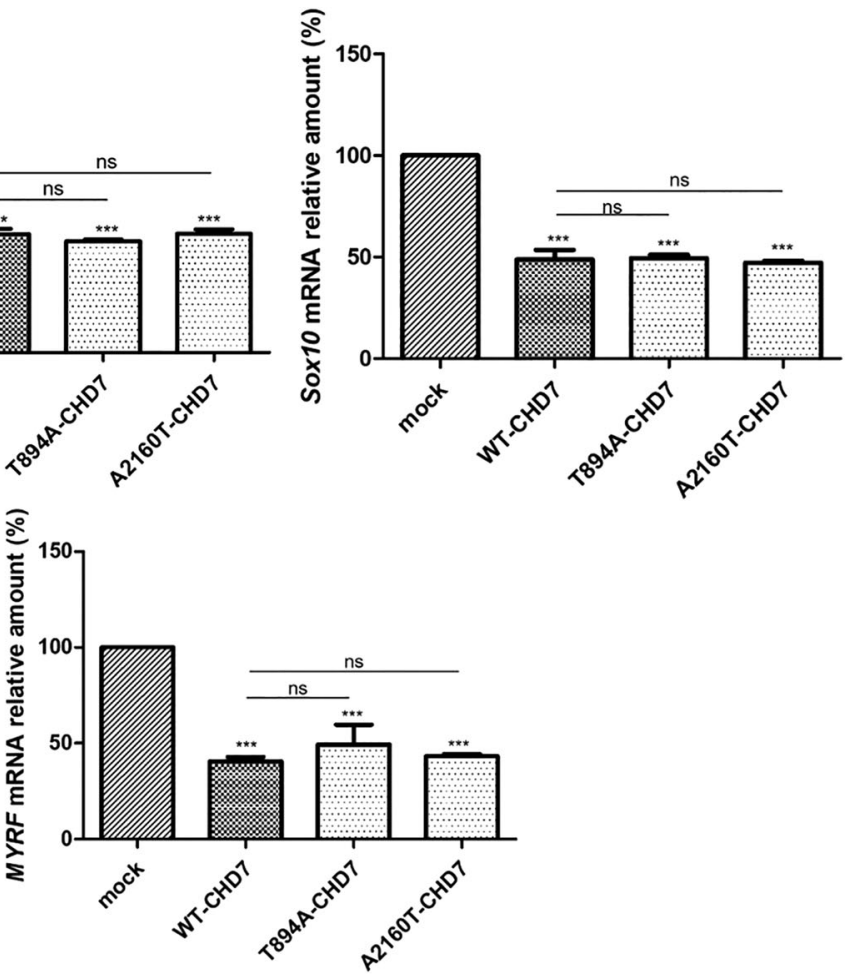

SOX4, SOX10, ID2, and MYRF. RNA amounts are displayed as an $n$ fold difference relative to the mock-transfected condition. Data represent means and standard deviation corresponding to at least three independent transfections. ns: nonsignificant, $* * * P<0.001$

then denaturation of the enzymes was carried out by heating at $100{ }^{\circ} \mathrm{C}$ for $2 \mathrm{~min}$. The cDNA solution was stored at $-20^{\circ} \mathrm{C}$. Finally, the cDNA was diluted $1 / 10$ for subsequent use in quantitative PCR.

\section{Real-time PCR}

A previous study showed that $45 \mathrm{~S}$ pre-rRNA expression level is positively correlated with CHD7 protein amount, in a colorectal cancer cell line and in mouse embryonic stem cells [14]. Another team described that, in dividing neural stem cells, CHD7 stimulates the expression of SOX4 and SOX11 genes via the remodeling of the chromatin region containing their promoters [25]. Furthermore, another study showed that CHD7 and Sox10 proteins co-operate to the target enhancers of several genes involved in myelination [26]. Among them, we chose as reporter genes MYRF and SOX10 itself, whose transcription was decreased in the spinal cord of $C H D 7$ conditional knockout mice, and ID2 which was upregulated. Therefore, we evaluated the transcription levels of $45 S$ rDNA, SOX4, SOX10, MYRF, and ID2 genes.

The sequence of oligonucleotides (Eurogentec) used for real-time PCR are listed in Supplementary Table 2. cDNA samples and oligonucleotides $(0.9 \mu \mathrm{M}$ for primers and 


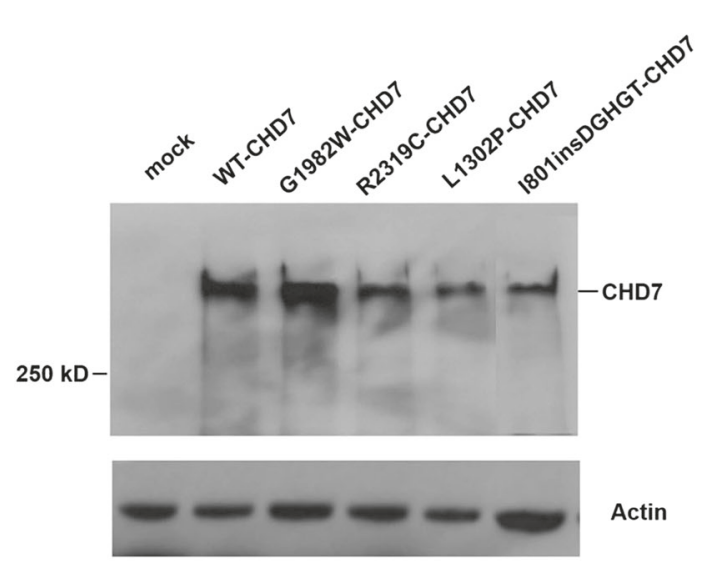

A

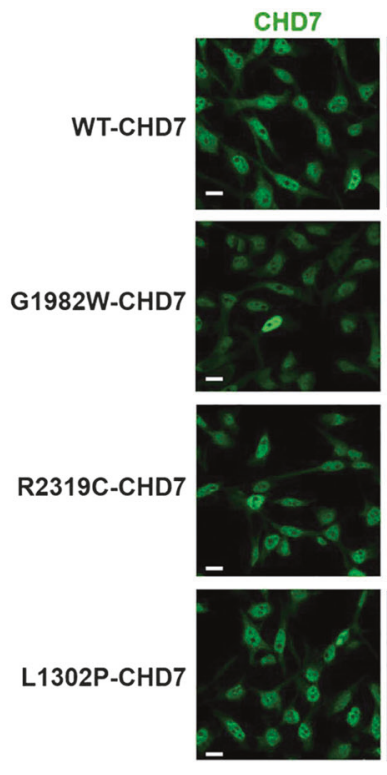

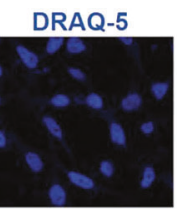
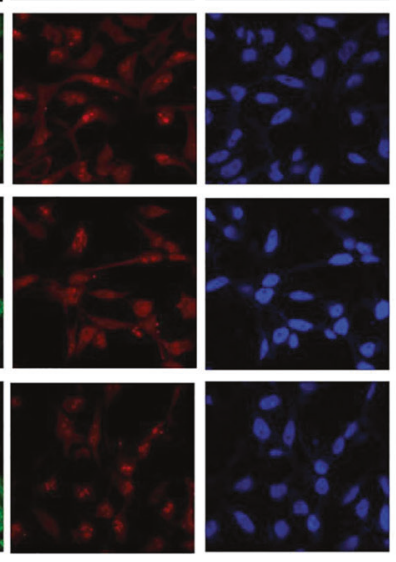

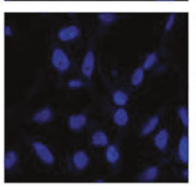

B
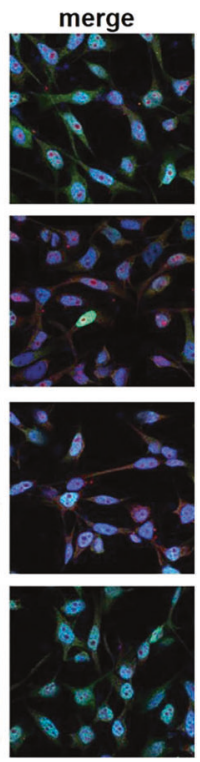

Fig. 3 Expression of wild type and variant forms of CHD7 protein in HeLa cells. a For each transfection, $100 \mu \mathrm{g}$ of protein lysates were separated by SDS-PAGE and analyzed by western blot with the antiCHD7 antibody, or anti-actin as a loading control. The expected molecular size of the CHD7 protein is $340 \mathrm{kD}$. b Nucleoplasmic localization of wild-type and variant forms of $\mathrm{CHD} 7$ protein expressed

$0.3 \mu \mathrm{M}$ for internal probe) were added to TaqMan Universal Master Mix (Applied Biosystems) in a final volume of $15 \mu \mathrm{L}$. PCR reactions were performed in triplicate on a 7500 Fast Real-Time PCR System (Applied Biosystems). A comparative threshold cycle $\left(\mathrm{C}_{\mathrm{T}}\right)$ was used to determine relative gene expression. For each gene, $\Delta \mathrm{C}_{\mathrm{T}}$ was calculated against the $G A P D H$ housekeeping gene. Hence, the value used to plot relative gene transcription level was determined using the expression $2^{-\Delta \Delta C T}$. RNA amounts were expressed as an $n$-fold difference relative to the mock-transfected condition. Results are displayed as the means and standard deviation corresponding to three independent transfections. To compare sets of data, we used the Student's $t$-test. All statistical tests were performed using GraphPad Prism version 7.0 software for Windows (Graphpad Software Inc., La Jolla, California, USA).

\section{Results}

\section{Assessment of wild-type CHD7 protein functionality}

First, we aimed to establish a functional test to evidence the functionality of normal CHD7 protein and subsequently to observe putative dysfunctions of CHD7 variants expressed in CS patients. CHD7 was proposed to regulate the transcription of numerous genes throughout development, by binding regulatory regions of the chromatin prior to in HeLa cells. Immunostaining was visualized using confocal microscopy. CHD7 protein was detected in green and nucleolin in red. DRAQ5 dye was used to color the nuclei (blue). We observed no colocalization between both proteins, suggesting that none of the CHD7 protein variants are nucleolar. Bar: $10 \mu \mathrm{m}$

remodeling. To investigate the functionality of $\mathrm{CHD7}$ alleles, we measured by qRT-PCR the consequence of CHD7 overexpression upon the RNA transcript amount for several genes whose transcription can be modulated by CHD7 according to the literature. We chose to quantify $45 \mathrm{~S}$ preribosomal RNA, and the mRNA of SOX4, SOX10, ID2, and MYRF genes, as explained in the Material and Methods section.

When we overexpressed WT-CHD7 in HeLa cells, the amount of RNA transcribed from these genes were downregulated, compared with mock-transfected cells (Fig. 2): the transcript amounts were reduced to $39.4 \pm 4.5 \%$ of control for $45 \mathrm{~S} r D N A, 60.5 \pm 1.5 \%$ for SOX4, $48.9 \pm 2.7 \%$ for $S O X 10,23.2 \pm 4.2 \%$ for $I D 2$ and $40.6 \pm 1.3 \%$ for $M Y R F$ gene. To validate these results, we applied our biological assay to two CHD7 protein variants, presenting one amino acid substitution but classified as nonpathogenic using GnomAD software predictive tool: p.(Thr894Ala) and p. (Ala2160Thr). We will refer below to these polymorphisms with the following abbreviations: T894A and A2160T, respectively. We generated plasmids encoding these variants using site-directed mutagenesis and, after transfection in HeLa cells, we first assessed the expression of these proteins by western blot and immunofluorescence experiments (Supplementary Fig. 2). Then, we quantified the transcript amounts of the five reporter genes: in both cases, mRNA amounts were significantly decreased compared with the mock transfection (Fig. 2). Moreover, no 


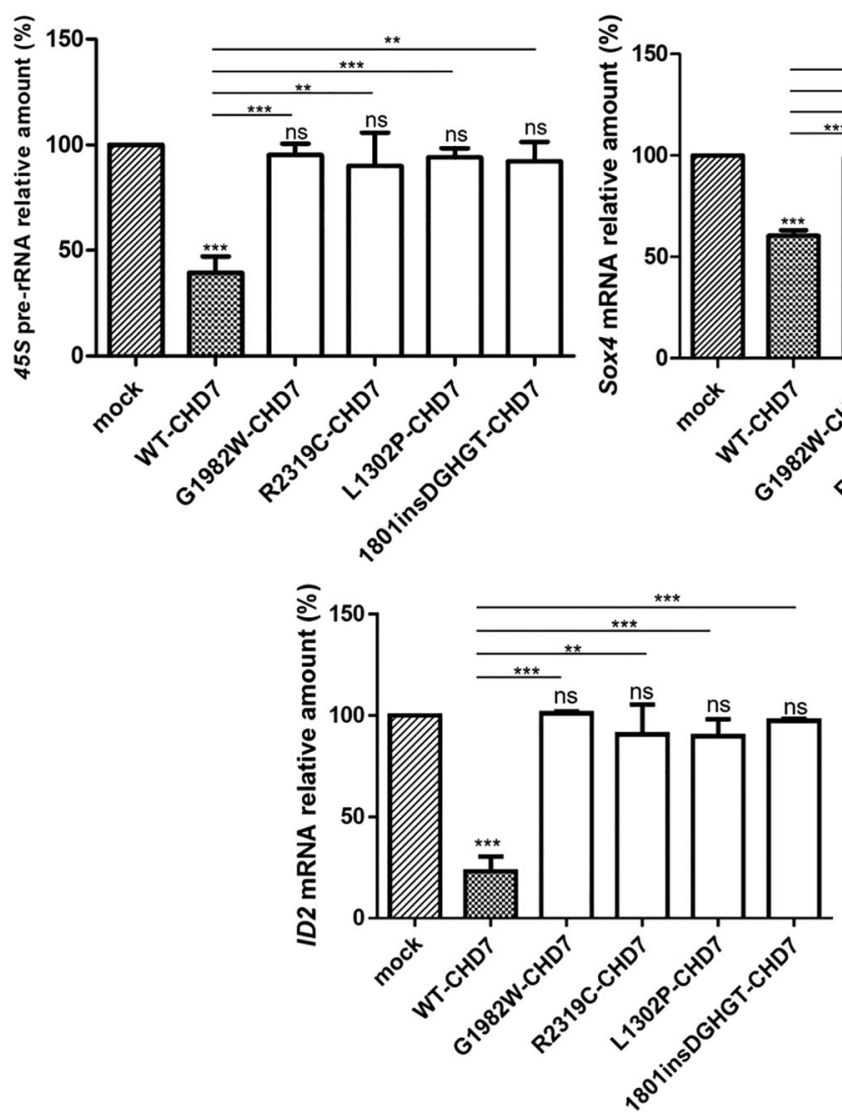

Fig. 4 Impact of G1982W, R2319C, L1302P, and 1801insDGHGTCHD7 variants expression upon the transcription of five reporter genes. After transfection of wild type or variant forms of CHD7, we performed qRT-PCR to evaluate the impact upon the transcription of genes regulated by CHD7: 45s rDNA, SOX4, SOX10, ID2, and MYRF.

statistically significant difference was observed compared with WT-CHD7 expression condition. Overall, we concluded that the reduction of the transcript amount of the reporter genes should result from the normal functionality of CHD7 protein in HeLa cells.

\section{Localization and functional assay of CHD7 missense variants}

Using site-directed mutagenesis, we generated plasmids encoding three $\mathrm{CHD} 7$ protein variants, corresponding to missense variations found in patients: p.(Gly1982Trp), p. (Arg2319Cys), and p.(Leu1302Pro). We will refer below to these variants with the following abbreviations: G1982W, R2319C, and L1302P, respectively. After plasmid transfection in HeLa cells, we evaluated the efficiency of CHD7 overexpression by western blot using CHD7 rabbit monoclonal antibody. First, we optimized the transfection conditions with WT-CHD7-encoding plasmid (not shown), then we analyzed the expression of the four CHD7 variants in HeLa cells (Fig. 3a). As expected, for WT and all variant
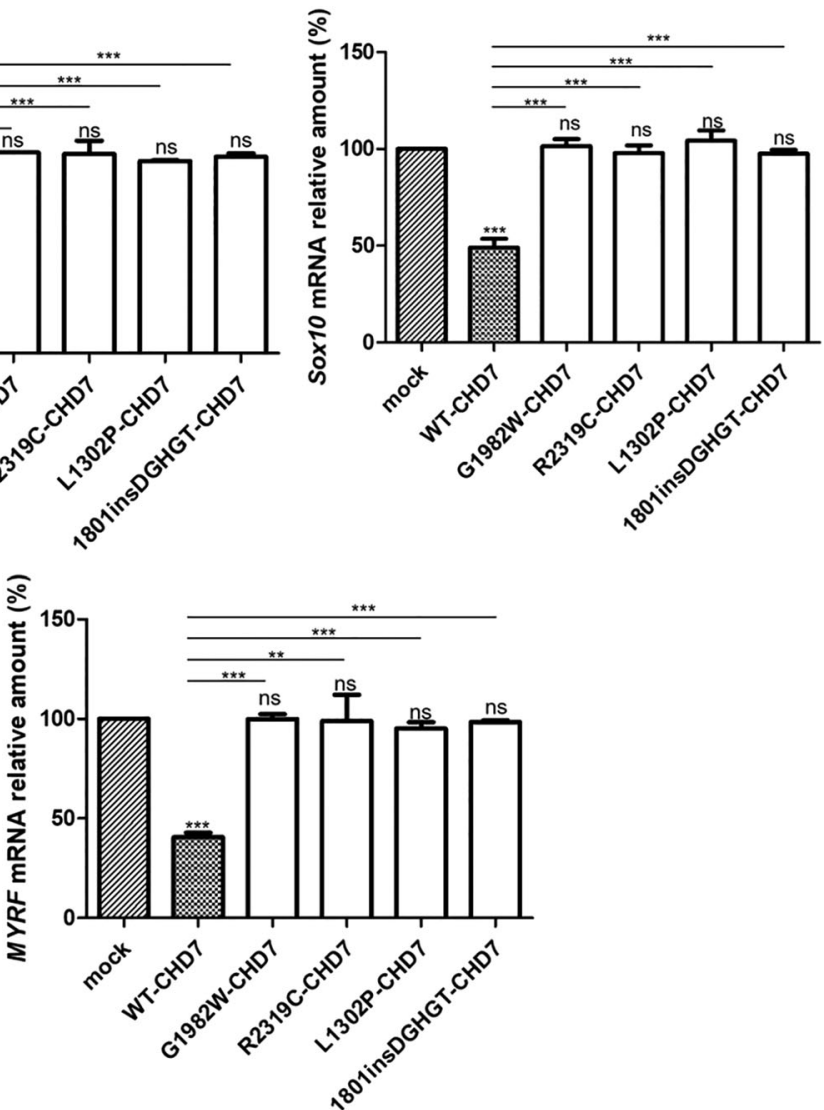

RNA amounts are displayed as an $n$-fold difference relative to the mock-transfected condition. Data represent means and standard deviation corresponding to at least three independent transfections. ns: nonsignificant, $* * P<0.01, * * * P<0.001$

forms of CHD7 protein, a specific protein band appeared at a position corresponding to a relative molecular mass of $\sim 340 \mathrm{kD}$. In parallel, the detection of $\beta$-actin protein was performed as a protein loading control. Hence, each of the plasmids that we used elicited the expression of the corresponding allele of $\mathrm{CHD} 7$ protein.

To investigate the subcellular localization of the different forms (WT or resulting from missense variations) of CHD7 protein expressed in HeLa cells, we performed immunofluorescent staining followed by confocal microscopy imaging. CHD7 localization has previously been assessed in the nucleoplasm and in the nucleolus $[14,26]$. Therefore, we performed double immunostaining against CHD7 and nucleolin, a protein expressed all over the nucleus but mostly concentrated in the nucleolus. We observed that the three variants, G1982W-, R2319C-, and L1302P-CHD7, exhibited the same localization as the WT form of the protein (Fig. 3b). In each case, CHD7 appeared localized exclusively in the nucleoplasm. The superposition with nucleolin staining highlights that, in our hands, CHD7 was excluded from the nucleoli. In several cells, the intensity of 
CHD7 staining appeared higher, but the localization remained confined to the nucleoplasm: therefore, higher overexpression level did not alter the observed localization. Moreover, the three amino acid substitutions found in patients had no impact upon CHD7 trafficking.

When we expressed WT-CHD7 in HeLa cells, the amounts of RNA transcribed from reporter genes were downregulated, compared with mock-transfected cells. By contrast, when we expressed either one of the three missense $C H D 7$ variant alleles, the transcript amount of each reporter gene was unaffected, compared with the mock-transfected control condition (Fig. 4). This result suggests that the normal ability of CHD7 protein to modify the transcription level of several genes was impaired by the three amino acid substitutions that we investigated.

\section{Functionality of CHD7 insertion variant}

We previously described the finding in several CS patients of the c.5405-17 G>A splicing alteration, localized in a hotspot of intronic variations [22]. We assessed by the minigene assay that it results in a fifteen intronic nucleotide insertion in the mRNA, presumably leading to the synthesis of a five-amino acid insertion in the protein, i.e., p. (His1801_Gly1802insAspGlyHisGlyThr). We will refer below to this protein variant with the abbreviation 1801insDGHGT.

We generated a plasmid encoding this variant, and we used it to transfect HeLa cells. We checked by western blot and immunofluorescence that it elicited overexpression of the protein (Fig. 3a and Supplementary Fig. 3). Then, we evaluated the impact of 1801insDGHGT-CHD7 overexpression upon the transcription of the five reporter genes: we observed that their mRNA level was not significantly different of the mock-transfection condition (Fig. 4). This suggests that the 1801insDGHGT-CHD7 protein variant is nonfunctional.

\section{Discussion}

Most cases of CHARGE syndrome result from alterations in one allele of the $C H D 7$ gene. A small proportion of these defects elicit changes in amino acid sequence, mostly missense variations. However, no biological assay is available to discriminate if an alteration in CHD7 amino acid sequence is a polymorphism or results in a dysfunctional protein. In this work, we describe a straightforward approach to evaluate the impact of amino acid sequence variations upon the functionality of CHD7 protein. We applied this method to four variants that we found in CHARGE patients: three amino acid substitutions,
G1982W, R2319C, and L1302P, and one five-amino acid insertion, 1801insDGHGT.

The G1982W variant was found in a patient with typical CHARGE syndrome. This substitution is in the SANT-like domain (Fig. 1), which is present in many subunits of chromatin remodeling complexes. Its precise function is unclear, but it could be involved in binding to DNA and histones [11]. The R2319C variant was identified in a case of partial CHARGE. This amino acid change lies in a CHD7 region whose sequence presents no homology with any consensus domain (Fig. 1). The third missense variant, L1302P, is a familial variation case that displays variable phenotypic manifestations. This substitution is localized in a helicase domain (Fig. 1), whose integrity may be essential to the normal function of CHD7.

The c.5405-17 G>A substitution lies in a recurrent hotspot of intronic variations. It results in the creation of a new acceptor splice site that can overcome the weakness of the natural acceptor site. This elicits the maturation of an mRNA encoding the 1801 insDGHGT-CHD7 protein variant, presenting a five-amino acid insertion between the helicase and the SANT domains. When we applied our functional assay to this variant, it appeared nonfunctional and thus disease-associated. However, this nucleotide substitution has been found in several unrelated families. As previously described, patients exhibited either atypical or typical CS with various combinations of clinical anomalies and intra-familial variability [22]. Indeed, a splicing defect can occur with various intensities according to the patients, leading to differences in variant protein amount during early development. This may account for the diversity of clinical features in patients presenting this variant.

Whatever the structural consequence of these changes in amino acid sequence upon CHD7 folding, we designed a simple functional assay based on CHD7 ability to regulate gene transcription. First, we checked the overexpression of wild type and variants form of CHD7 by western blot and immunofluorescence. Several studies concluded that CHD7 localized throughout the nucleus but was more concentrated in the nucleoli $[14,26]$. However, in our hands, WT-CHD7, either endogenous or overexpressed, always appeared preferentially localized in the nucleoplasm of HeLa cells. Moreover, we obtained similar results in HEK293 (human epithelial kidney) or SH-SY5Y (human neuroblast) cell lines, when expressing $\mathrm{N}$-tagged, C-tagged, or untagged forms of WT-CHD7 (data not shown). In HeLa cells, we observed that the three variant forms of CHD7 exhibit the same localization compared with the wild-type. This suggests that these changes in CHD7 amino acid sequence have no consequence upon the intracellular trafficking of the protein.

To compare the functionality of the three variants with that of WT-CHD7, we quantified the consequence of CHD7 
overexpression in HeLa cells upon the transcript amounts of genes whose transcription was published to be regulated by CHD7. When we transiently expressed WT-CHD7 in HeLa cells, the transcription of the five reporter genes was downregulated compared with mock-transfected cells. With the overexpression approach that we used, the modulation of transcription elicited by CHD7 appeared sometimes contradictory with previously published data obtained in various ex vivo or in vivo models, in which $\mathrm{CHD7}$ was either overexpressed or depleted. However, in a transformed cell line like HeLa cells, the chromatin packing status and epigenetic tagging may differ largely compared with these models.

Anyway, when we expressed any of the four CHD7 variants, the transcription levels of the five reporter genes were nonsignificantly different of the control levels. This suggests that these CHD7 variants have lost their functionality of chromatin remodeller and gene transcription regulator. Hence, these amino acid changes are likely lossof-function variants, leading to haploinsufficiency in patients. For amino acid changes R2319C and L1302P, our data confirm the in silico predictions that they should be classified as class 5 (disease-causing) variations. As for G1982W, we propose that this variation should be upgraded from class 3 (uncertain significance) to class 4 (probably affecting function). In conclusion, we have developed a novel and straightforward assay to assess accurately the functional impact of CHD7 amino acid sequence variants. Particularly, applying this approach at a larger scale will allow classifying many of the $C H D 7$ variants that have been listed, but whose significance in CS remains unknown.

Acknowledgements We thank Anne Cantereau (STIM-CNRS ERL 7003, Poitiers, France) for technical assistance for confocal microscopy. This research was funded by the University of Poitiers, France. We also thank Indonesia Endowment Fund for Education (LPDP), Ministry of Finance of the Republic of Indonesia, for financial support.

Funding Supported by Université de Poitiers, Poitiers, France.

\section{Compliance with ethical standards}

Conflict of interest The authors declare that they have no conflict of interest.

Publisher's note: Springer Nature remains neutral with regard to jurisdictional claims in published maps and institutional affiliations.

\section{References}

1. Hall BD. Choanal atresia and associated multiple anomalies. J Pediatr. 1979;3:395-8.

2. Hittner HM, Hirsch NJ, Kreh GM, Rudolph AJ. Colobomatous microphthalmia, heart disease, hearing loss, and mental retardation-a syndrome. J Pediatr Ophthalmol Strabismus 1979;16:122-8.
3. Pagon RA, Graham JM, Zonana J, Yong SL. Coloboma, congenital heart disease, and choanal atresia with multiple anomalies: CHARGE association. J Pediatr. 1981;99:223-7.

4. Verloes A. Updated diagnostic criteria for CHARGE syndrome: a proposal. Am J Med Genet. 2005;133A:306-8.

5. Issekutz KA, Graham JM, Prasad C, Smith IM, Blake KD. An epidemiological analysis of CHARGE syndrome: preliminary results from a Canadian study. Am $J$ Med Genet. 2005;133A:309-17.

6. Vissers LE, van Ravenswaaij CM, Admiraal R, Hurst JA, de Vries $\mathrm{BB}$, Janssen IM, et al. Mutations in a new member of the chromodomain gene family cause CHARGE syndrome. Nat Genet. 2004;36:955-7.

7. Janssen N, Bergman JEH, Swertz MA, Tranebjaerg L, Lodahl M, Schoots J, et al. Mutation update on the CHD7 gene involved in CHARGE syndrome. Hum Mutat. 2012;33:1149-60.

8. Jongmans MC, van Ravenswaaij-Arts CM, Pitteloud N, Ogata T, Sato N, Claahsen-van der Grinten HL. et al. CHD7 mutations in patients initially diagnosed with Kallmann syndrome-the clinical overlap with CHARGE syndrome. Clin Genet. 2009;75:65-71.

9. Gennery AR, Slatter MA, Rice J, Hoefsloot LH, Barge D, McLean-Tooke A, et al. Mutations in CHD7 in patients with CHARGE syndrome cause T-B + natural killer cell + severe combined immune deficiency and may cause Omenn-like syndrome. Clin Exp Immunol. 2008;153:75-80.

10. Micucci JA, Sperry ED, Martin DM. Chromodomain helicase DNA-binding proteins in stem cells and human developmental diseases. Stem Cells Dev. 2015;24:917-26.

11. Basson MA, van Ravenswaaij-Arts C. Functional insights into chromatin remodelling from studies on CHARGE syndrome. Trends Genet. 2015;31:600-11.

12. Schnetz MP, Bartels CF, Shastri K, Balasubramanian D, Zentner GE, Balaji R, et al. Genomic distribution of CHD7 on chromatin tracks H3K4 methylation patterns. Genome Res. 2009;19:590-601.

13. Zentner GE, Hurd EA, Schnetz MP, Handoko L, Wang C, Wang $\mathrm{Z}$, et al. CHD7 function in the nucleolus as a positive regulator of ribosomal RNA biogenesis. Hum Mol Genet. 2010;19:3491-501.

14. Bergman JE, Janssen N, van der Sloot AM, de Walle HE, Schoots $\mathrm{J}$, Rendtorff ND, et al. A novel classification system to predict the pathogenic effects of CHD7 missense variants in CHARGE syndrome. Hum Mutat. 2012;33:1251-60.

15. Butcher DT, Cytrynbaum C, Turinsky AL, Siu MT, InbarFeigenberg M, Mendoza-Londono R, et al. CHARGE and Kabuki syndromes: gene-specific DNA methylation signatures identify epigenetic mechanisms linking these clinically overlapping conditions. Am J Hum Genet. 2017;100:773-88.

16. Aref-Eshghi E, Rodenhiser DI, Schenkel LC, Lin H, Skinner C, Ainsworth P, et al. Genomic DNA methylation signatures enable concurrent diagnosis and clinical genetic variant classification in neurodevelopmental syndromes. Am J Hum Genet. 2018;102:156-74.

17. Bouazoune K, Kingston RE. Chromatin remodeling by the CHD7 protein is impaired by mutations that cause human development disorders. Proc Natl Acad Sci USA. 2012;47:19238-43.

18. Bilan F, Legendre M, Charraud V, Manière B, Couet D, GilbertDussardier B, et al. Complete screening of 50 patients with CHARGE syndrome for anomalies in the CHD7 gene using a denaturing high-performance liquid chromatography-based protocol: new guidelines and a proposal for routine diagnosis. J Mol Diagn. 2012;14:46-55.

19. Legendre M, Abadie V, Attié-Bitach T, Philip N, Busa T, Bonneau D, et al. Phenotype and genotype analysis of a French cohort of 119 patients with CHARGE syndrome. Am J Med Genet C Semin Med Genet. 2017;175:417-30. 
20. Richards S, Aziz N, Bale S, Bick D, Das S, Gastier-Foster J, et al. ACMG Laboratory Quality Assurance Committee: standards and guidelines for the interpretation of sequence variants: a joint consensus recommendation of the American College of Medical Genetics and Genomics and the Association for Molecular Pathology. Genet Med. 2015; 17:405-24.

21. Legendre M, Rodriguez-Ballesteros M, Rossi M, Abadie V, Amiel J, Revencu N, et al. CHARGE syndrome: a recurrent hotspot of mutations in CHD7 IVS25 analyzed by bioinformatic tools and minigene assays. Eur J Hum Genet. 2018;26:287-92.

22. Liu H, Naismith JH. An efficient one-step site-directed deletion, insertion, single and multiple-site plasmid mutagenesis protocol. BMC Biotechnol. 2008;8:91-100.
23. Bilan F, Thoreau V, Nacfer M, Dérand R, Norez C, Cantereau A, et al. Syntaxin 8 impairs trafficking of cystic fibrosis transmembrane conductance regulator (CFTR) and inhibits its channel activity. J Cell Sci. 2004;117:1923-35.

24. Feng W, Khan MA, Bellvis P, Zhu Z, Bernhardt O, Herold-Mende $\mathrm{C}$, et al. The chromatin remodeler $\mathrm{CHD} 7$ regulates adult neurogenesis via activation of SoxC transcription factors. Cell Stem Cell. 2013;13:62-72.

25. He D, Marie C, Zhao C, Kim B, Wang J, Deng Y, et al. Chd7 cooperates with Sox10 and regulates the onset of CNS myelination and remyelination. Nat Neurosci. 2016;19:678-89.

26. Kita Y, Nishiyama M, Nakayama KI. Identification of $\mathrm{CHD}_{\mathrm{S}}$ as a novel splicing variant of CHD7 with functions similar and antagonistic to those of the full-length $\mathrm{CHD}_{\mathrm{L}}$. Genes Cells. 2012;17:536-47. 